\title{
Phenome-wide association study (PheWAS) in EMR-linked pediatric cohorts, genetically links PLCL1 to speech language development and IL5-IL13 to Eosinophilic Esophagitis
}

\author{
Bahram Namjou 1,2*, Keith Marsolo 2,3 , Robert J. Caroll ${ }^{4}$, Joshua C. Denny ${ }^{4,5}$, Marylyn D. Ritchie ${ }^{6}$, \\ Shefali S. Verma ${ }^{6}$, Todd Lingren ${ }^{2,3}$, Aleksey Porollo ${ }^{1,2,3}$, Beth L. Cobb ${ }^{1}$, Cassandra Perry ${ }^{7}$, \\ Leah C. Kottyan ${ }^{1,2,8}$, Marc E. Rothenberg ${ }^{8}$, Susan D. Thompson ${ }^{1,2}$, Ingrid A. Holm ${ }^{9}$, Isaac S. Kohane ${ }^{10}$ \\ and John B. Harley ${ }^{1,2,11}$
}

\author{
Center for Autoimmune Genomics and Etiology, Cincinnati Children's Hospital Medical Center, Cincinnati, OH, USA \\ ${ }^{2}$ College of Medicine, University of Cincinnati, Cincinnati, OH, USA \\ ${ }^{3}$ Division of Biomedical Informatics, Cincinnati Children's Hospital Medical Center, Cincinnati, OH, USA \\ ${ }^{4}$ Department of Biomedical Informatics, Vanderbilt University School of Medicine, Nashville, TN, USA \\ ${ }^{5}$ Department of Medicine, Vanderbilt University School of Medicine, Nashville, TN, USA \\ ${ }^{6}$ Center for Systems Genomics, The Pennsylvania State University, Philadelphia, PA, USA \\ 7 Division of Genetics and Genomics, Boston Children's Hospital, Boston, MA, USA \\ ${ }^{8}$ Division of Allergy and Immunology, Department of Pediatrics, Cincinnati Children's Hospital Medical Center, Cincinnati, OH, USA \\ ${ }^{9}$ Division of Genetics and Genomics, Department of Pediatrics, The Manton Center for Orphan Disease Research, Harvard Medical School, Boston Children's \\ Hospital, Boston, MA, USA \\ ${ }^{10}$ Children's Hospital Informatics Program, Center for Biomedical Informatics, Harvard Medical School, Boston, MA, USA
}

$"$ U.S. Department of Veterans Affairs Medical Center, Cincinnati, OH, USA

\section{Edited by:}

Mariza De Andrade, Mayo Clinic, USA

\section{Reviewed by:}

Andrew Skol, University of Chicago, USA

Albert Vernon Smith, Icelandic Heart Association, Iceland

Shelley Cole, Texas Biomedical

Research Institute, USA

\section{*Correspondence:}

Bahram Namjou, Cincinnati Children's Hospital Medical Center, 3333 Burnet Avenue, Cincinnati, $\mathrm{OH}$ 45229, USA

e-mail: bahram.namjou@cchmc.org
Objective: We report the first pediatric specific Phenome-Wide Association Study (PheWAS) using electronic medical records (EMRs). Given the early success of PheWAS in adult populations, we investigated the feasibility of this approach in pediatric cohorts in which associations between a previously known genetic variant and a wide range of clinical or physiological traits were evaluated. Although computationally intensive, this approach has potential to reveal disease mechanistic relationships between a variant and a network of phenotypes.

Method: Data on 5049 samples of European ancestry were obtained from the EMRs of two large academic centers in five different genotyped cohorts. Recently, these samples have undergone whole genome imputation. After standard quality controls, removing missing data and outliers based on principal components analyses (PCA), 4268 samples were used for the PheWAS study. We scanned for associations between 2476 single-nucleotide polymorphisms (SNP) with available genotyping data from previously published GWAS studies and 539 EMR-derived phenotypes. The false discovery rate was calculated and, for any new PheWAS findings, a permutation approach (with up to 1,000,000 trials) was implemented.

Results: This PheWAS found a variety of common variants (MAF > 10\%) with prior GWAS associations in our pediatric cohorts including Juvenile Rheumatoid Arthritis (JRA), Asthma, Autism and Pervasive Developmental Disorder (PDD) and Type 1 Diabetes with a false discovery rate $<0.05$ and power of study above $80 \%$. In addition, several new PheWAS findings were identified including a cluster of association near the NDFIP1 gene for mental retardation (best SNP rs 10057309, $p=4.33 \times 10^{-7}, O R=1.70,95 \% \mathrm{Cl}=1.38-2.09$ ); association near PLCL1 gene for developmental delays and speech disorder [best SNP rs1595825, $p=1.13 \times 10^{-8}, O R=0.65(0.57-0.76)$ ]; a cluster of associations in the IL5-IL13 region with Eosinophilic Esophagitis (EoE) [best at rs12653750, $p=3.03 \times 10^{-9}$, $O R=1.7395 \% \mathrm{Cl}=(1.44-2.07)]$, previously implicated in asthma, allergy, and eosinophilia; and association of variants in GCKR and JAZF1 with allergic rhinitis in our pediatric cohorts [best SNP rs780093, $p=2.18 \times 10^{-5}, O R=1.39,95 \% \mathrm{Cl}=(1.19-1.61)$ ], previously demonstrated in metabolic disease and diabetes in adults.

Conclusion: The PheWAS approach with re-mapping ICD-9 structured codes for our European-origin pediatric cohorts, as with the previous adult studies, finds many previously reported associations as well as presents the discovery of associations with potentially important clinical implications. 


\section{INTRODUCTION}

Phenome-wide association study (PheWAS) is a relatively new genomic approach to link clinical conditions with published variants (Denny et al., 2010). The concept, although not new, was originally applied to genomic research by the eMERGE (electronic MEdical Records and GEnomics) network, which is in a unique position to access tens of thousands of Electronic Medical Records (EMR) linked to ICD-9 codes in structured data. Multiple eMERGE PheWAS results have been published that primarily address adult cohorts (Denny et al., 2011, 2013). The phenotypic data used in PheWAS may include ICD-9 codes, epidemiologic data in health surveys, biomarkers, intermediate or quantitative traits (Pendergrass et al., 2011, 2013; Neuraz et al., 2013; Liao et al., 2014). By virtue of this inclusive approach, new hypotheses may be generated that provide insight into genetic architecture of complex traits. Challenges with PheWAS include multiple test corrections across the thousands of phenotypes tested and autocorrelation of some of the phenotypes. Nevertheless, novel robust insights have resulted from PheWAS, for example, genetic association findings with heart rate variability are notable (Ritchie et al., 2013).

PheWAS combines multiple phenotypes from previous GWAS, and identify common SNPs affecting different traits. In this study, we used this approach to evaluate whether known GWAS variants identified in adult diseases can be also identified in children using two EMR-linked pediatric datasets from eMERGE. PheWAS in pediatrics is particularly important because it not only assesses the effect of early age of onset on many established adult-GWAS loci, but also may provide insights into how a primary phenotype during child development develops into one or more diseases in adulthood. A priori, there are several reasons that in principle might make a pediatric PheWAS more challenging. These include the change in heritability with age for several traits (St Pourcain et al., 2014), the flux in the recommendations for pediatric monitoring for traits that are routinely measured in adults (Gidding, 1993; Klein et al., 2010) and the use of cross-sectional standardization rather than longitudinal standardization of developmental traits such as height (Tiisala and Kantero, 1971).

To determine whether robust association signals would be present in the context of these challenges, we conducted the first PheWAS study in pediatrics on our available samples. We successfully translated 93,724 specific ICD-9 diagnostic codes into 1402 distinct PheWAS code groups and 14 major disease concept paths and evaluated 2481 previously published variants. After quality control, only 2476 genetic variants were analyzed in 539 diseases in the two pediatric sites. Finally we replicated 24 genetic variants and identified 14 new possible associations confirming our hypothesis. Our primary results highlight the utility of an EMR-based PheWAS approach as a new line of investigation for discovery of genotype-phenotype associations in pediatrics.

\section{MATERIALS AND METHODS STUDY SUBJECTS}

Protocols for this study were approved by the Institutional Review Boards (IRBs) at the institutions where participants were recruited. All study participants provided written consent prior to study enrolment; consent forms were obtained at each location under IRB guidelines. Children and teens, aged through 19 years old were included. The EMR-linked pediatric emerge cohorts consist of 4560 subjects from Cincinnati Children's Hospital Medical Center (CCHMC) and 1000 subjects from Boston Children's Hospital (BCH). Only those self-reported to have European ancestry were selected for this study (Table 1).

\section{SNP PRIORITIZATION}

We limit our investigation to particular genetic variants: First, we obtained the list of all previously published SNPs from different public domain databases including The National Human Genome Research Institute (NHGRI) catalog of published Genome-Wide Association Studies (http://www.genome. gov/gwastudies), Genetic Association of Complex Diseases and Disorders (GAD, http://geneticassociationdb.nih.gov), the UCSC Genome Browser database (UCSC, http://genome.ucsc. edu/), Online Mendelian Inheritance in Man (OMIM, http:// www.omim.org/), and PharmGKB (pharmgkb, https://www. pharmgkb.org). After linking this collection to PubMed reference numbers, only those with at least one reported of positive associations were selected regardless of the previously observed $p$ values or number of publications. In addition, all downloaded databases were current at the time of this submission. From the filtered variants, 2476 variants were available and assessed in our clean, post-imputation genotyping dataset for analysis.

\section{GENOTYPING AND STATISTICAL ANALYSES}

High throughput SNP genotyping was carried out previously in $\mathrm{CCHMC}$ and $\mathrm{BCH}$ using Illumina ${ }^{\mathrm{TM}}$ or Affymetrix ${ }^{\mathrm{TM}}$ platforms, as previously described (Namjou et al., 2013). Quality control (QC) of the data was performed before imputation. In each genotyped cohort, standard quality control criteria were met and single nucleotide polymorphisms (SNPs) were removed if (a) $>5 \%$ of the genotyping data was missing, (b) out of HardyWeinberg equilibrium (HWE, $p<0.001$ ) in controls, or a minor allele frequency $(\mathrm{MAF})<1 \%$. Samples with call rate $<98 \%$ were excluded.

Recently all eMERGE cohorts have also undergone whole genome imputation. The details of these procedures are available in this issue of Frontiers in Genetics (Setia et al., 2014). Briefly, the imputation pipeline was implemented using IMPUTE2 program and the publicly available 1000-Genomes Project as the reference haplotype panel composed of 1092 samples (release version 2 from March 2012 of the 1000 Genomes Project Phase I, ftp://ftp-trace.ncbi.nih.gov/1000genomes/ftp/ release/20110521) (Howie et al., 2011). The eMERGE imputed data provided to us were already filtered, i.e., imputed data with a threshold of 0.90 for the genotype posterior probability and with a IMPUTE2 info score $>0.7$ (Howie et al., 2011). Principle component analysis (PCA) performed to identify outliers and hidden population structure using EIGENSTRAT (Price et al., 2006). The first two principle components explained most of the variance and were retained and used as covariates during the association analysis in order to adjust for population stratification. In addition, 14 outlier samples were removed. To illustrate the overall inflation rate a phenotype with sufficient number of cases and 
Table 1 | The demographic distribution of the European ancestry population (CCHMC-BCH).

\begin{tabular}{|c|c|c|c|c|c|}
\hline & Cohort names & \#Europeans & M/F & Mean age $(95 \% \mathrm{Cl})$ & Array \\
\hline & Cytogenetics & 609 & $373 / 236$ & $7.18(6.73-7.63)$ & Illumina-Omni-1 \\
\hline & $\mathrm{EoE}^{\dagger}$ & 543 & $394 / 149$ & $12.27(11.70-12.67)$ & Illumina-Omni-5 \\
\hline & $J \mid A^{\ddagger}$ & 488 & $101 / 387$ & $13.70(13.13-14.23)$ & Affymetrix-6 \\
\hline Total & & 4268 & $2403 / 1865$ & $11.52(11.16-11.91)$ & \\
\hline
\end{tabular}

${ }^{*} B C H$, Boston Children's Hospital; ${ }^{* *} C C H M C$, Cincinnati Children's Hospital Medical center; $t$, Eosinophilic Esophagitis (EoE) cohorts; $\neq$, Juvenile Idiopathic Arthritis cohorts (JIA). The details of platforms used have been described elsewhere (Namjou et al., 2013).

controls has been selected (autism) and the inflation of $\lambda=1.03$ was obtained.

Next, from our prioritized SNP list mentioned above, 2481 variants were available. Five of these SNPs had a site-specific effect with either CCHMC or BCH $\left(p<10^{-5}\right.$ for the difference between sites) and were removed from final analyses. For each phenotype, logistic regression was performed between cases and control adjusted for two principal components using PLINK (Purcell et al., 2007). To investigate whether either the phenotype or the genotype has an effect on the outcome variable, we perform phenotypic and genotypic conditional analyses, controlling for the effect of a specific SNP or phenotype. After pruning of highly correlated SNPs $\left(r^{2}>0.5\right)$, we used false discovery rate (FDR) methods to correct for multiple testing using the Benjamini-Hochberg procedure implemented in PLINK (Purcell et al., 2007). As a result of $\mathrm{LD}$ pruning 1828 independent variants were used for the purpose of FDR estimation. $Q$ values correspond to the proportion of false positives among the results. Thus, $Q$ values less than 0.05 signify less than $5 \%$ of false positives and are accepted as a measure of significance (FDR $<0.05)$ in this study. For any novel PheWAS findings, an adaptive permutation approach was performed using a sample randomization strategy in which case and control labels were permuted randomly (with up to $1,000,000$ trials) in order to obtain empirical $p$ values [PLINK (Purcell et al., 2007)]. We also report previous known effects that only produce suggestive findings in our study $(0.05<p<0.001)$. Sample size and power calculations based on the size effect and risk allele frequency were estimated using QUANTO (Gauderman and Morrison, 2006). To graphically display results, LocusZoom was used (Pruim et al., 2010).

\section{PHENOTYPING}

A phenome-wide association analysis (PheWAS) was performed in which presence or absence of each PheWAS code [mapped from translated ICD-9 codes as per Carroll et al., 2014)] were considered as a binary phenotype. The per-patient ICD-9 codes were obtained from the i2b2 Research Patient Data Warehouse at $\mathrm{CCHMC}$ and $\mathrm{BCH}$. Also, these PheWAS codes were used to define comparison control groups by excluding the PheWAS case- code and those closely related to them in the ICD-9 hierarchy. Control groups for Crohn's Disease (CD), for instance, excluded CD, ulcerative colitis, and several other related gastrointestinal complaints. Similarly, control groups for myocardial infarction excluded patients with myocardial infarctions, as well as angina and other evidence of ischemic heart disease. The current PheWAS map and PheWAS script written in $\mathrm{R}$ is available [http://phewascatalog.org, (Carroll et al., 2014)]. In this study, subgroups of European cases with more than 20 samples were selected for PheWAS association study (539 subgroups) and the available published SNPs that passed quality controls were evaluated. The case cohorts for the two phenotypes of Juvenile Idiopathic Arthritis (JIA) and Eosinophilic Esophagitis (EoE) have both been previously published as parts of larger phenotype specific studies (Rothenberg et al., 2010; Thompson et al., 2012; Hinks et al., 2013). The origin of all case records is presented in Table 1. In this study, Juvenile Onset Rheumatoid Arthritis (JRA) is identified by ICD-9 codes and designated as JRA; when the criteria for Juvenile Idiopathic Arthritis (JIA) were applied in the studies of others (Thompson et al., 2012), then this phenotype was referred to as JIA.

\section{RESULTS}

In this study only European ancestry was included in the analysis to avoid potential bias induced by ancestry. The demographic distribution of the European ancestry population under study (Table 2) had 93,724 specific ICD-9 diagnostic codes representing 1402 distinct PheWAS code groups and 14 major disease concept paths. The frequencies of concept path hierarchy of the ontology (Figure 1) show the neuropsychiatric concept path as the most frequent and neoplastic and infection paths as the least frequent.

\section{Replication of existing associations using PheWAS}

We compared SNPs with previous GWAS-reports and present association findings (FDR-q < 0.05) after corrected for population stratification and standard quality control (Table 2).

First, for the two phenotypes of JRA and EoE samples overlap largely with those previously reported phenotype specific GWAS study (Rothenberg et al., 2010; Thompson et al., 2012; Kottyan et al., 2014). We reproduced the major findings of those publications using different methodology. For JRA, association with PTPN22 is a consistent finding. As expected, we replicated a previous report of association of PTPN22 at non-synonymous coding SNP rs2476601 with this phenotype and with the same direction 


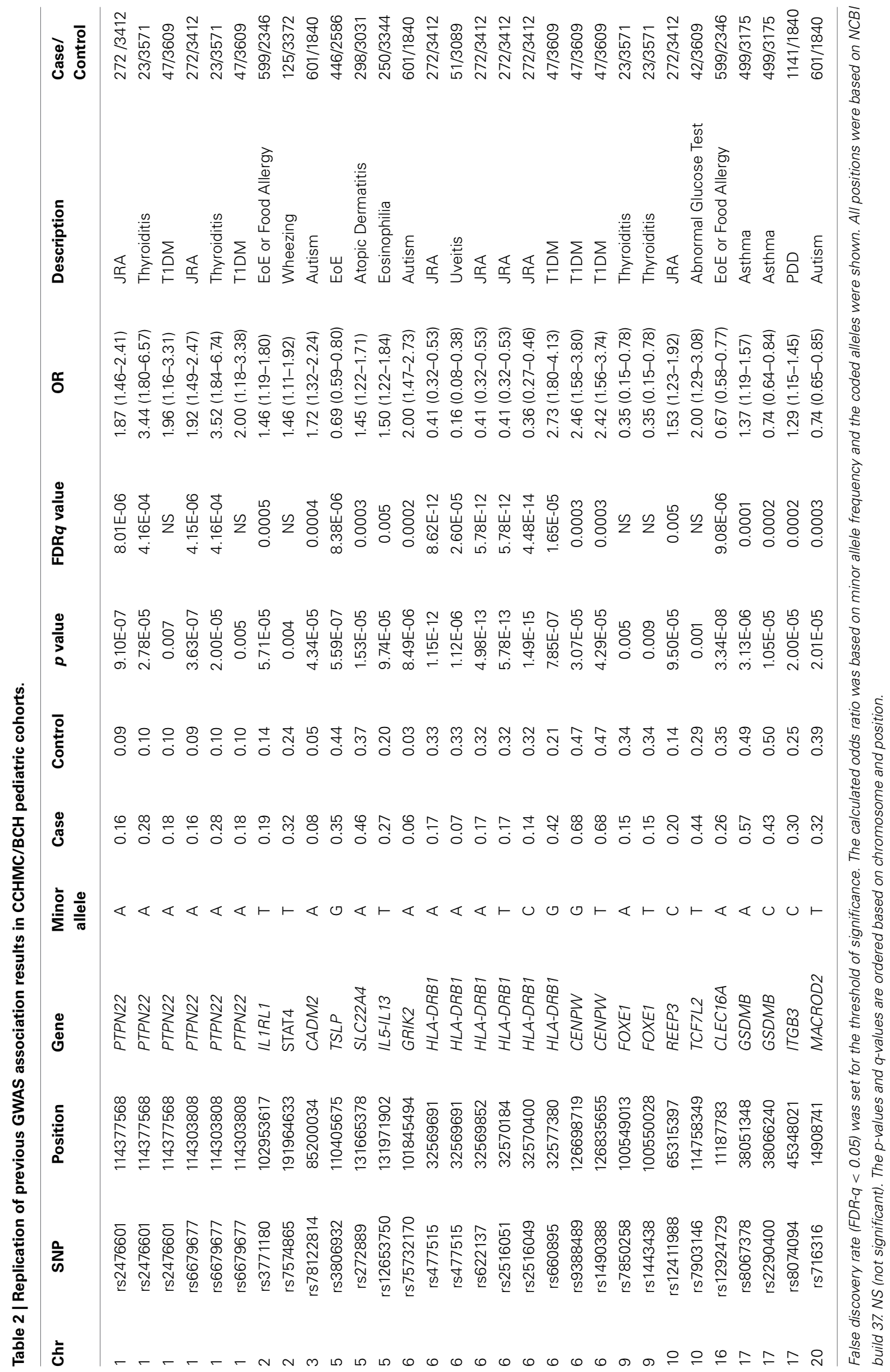




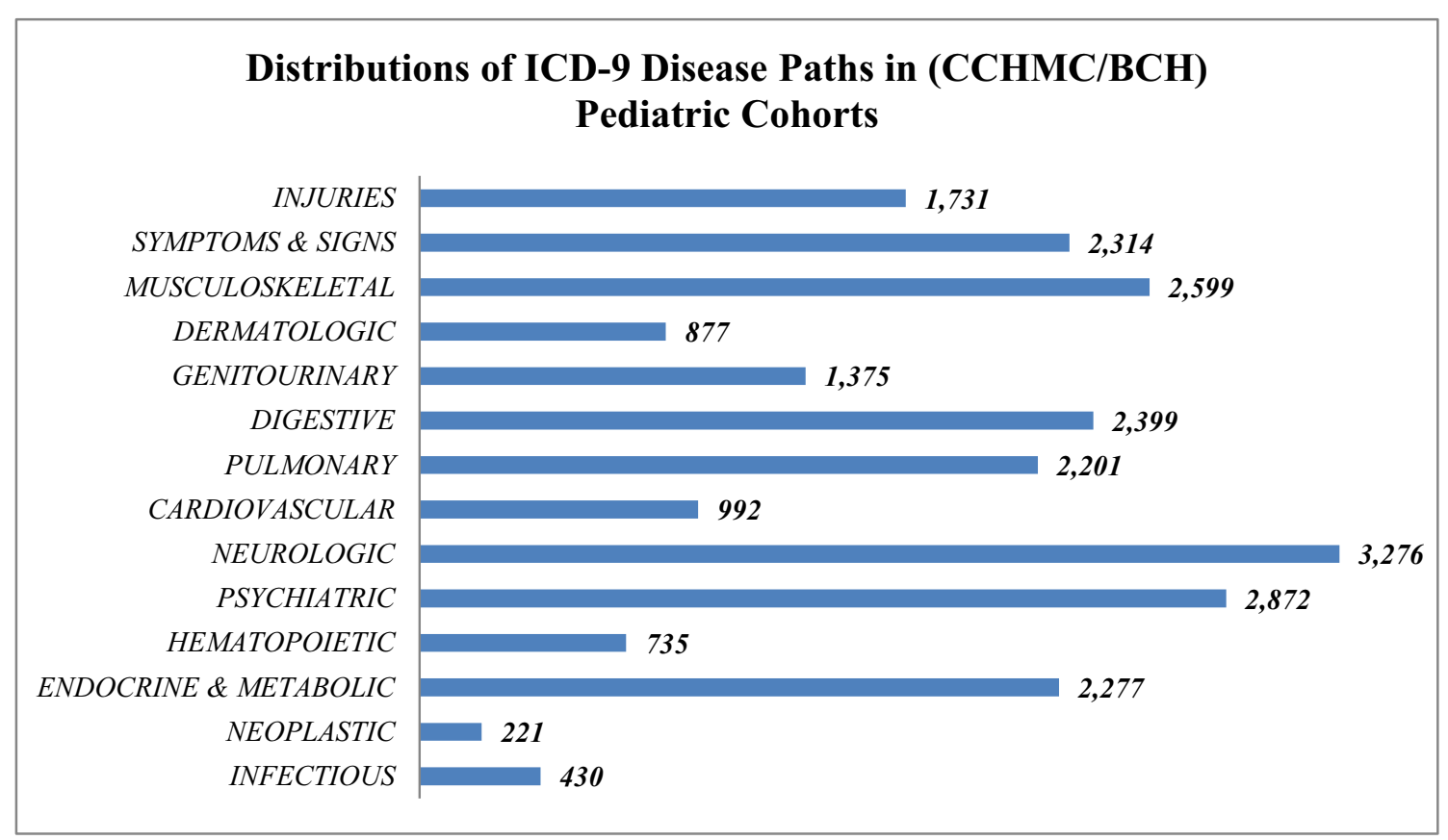

FIGURE 1 | Frequency and distribution of 14 major ontology concept path categories from CCHMC/BCH European pediatric cohorts.

of allele frequency, $\left(p=9.10 \times 10^{-7}, O R=1.87,95 \%\right.$ CI $1.46-$ 2.40). The SNP in proxy (rs6679677, $\left.r^{2}=1\right)$ also produced a similar result (Table 2). In our cohorts, variants in PTPN22 are also associated with thyroiditis as well as Type 1 diabetes mellitus (T1DM), consistent with previous reports and despite low sample size (Table 2) (Plenge et al., 2007; Todd et al., 2007; Lee et al., 2011). From these three known associations of PTPN22, i.e., JRA, T1DM, and thyroiditis, the largest magnitude of the association is with pediatric onset thyroiditis (Table 2, OR $=3.52$ 95\%CI $1.84-6.75)$.

For JRA, multiple loci in the HLA region were also associated at the level of $p<10^{-12}$ including rs477515 and rs2516049 near HLA-DRB1 (Table 2). Of note, the size effect of HLA related SNPs, were highest for those with coexisting uveitis (best SNP rs477515, $O R=6.5,95 \% \mathrm{CI}=2.73-15.68$ for the risk allele, Table 2). In addition, for JRA, another previously published association (rs12411988 in REEP3) was also found and with the same size effect as previously described $(O R=1.53)$ (Table 2) (Thompson et al., 2012).

Furthermore, with regard to EoE traits, we also replicated previous major finding of association of SNP rs3806932 located at the vicinity of the TSLP gene at $5 q 22$ region $[p=5.59 \times 10-7$, $O R=0.69(95 \% \mathrm{CI}=0.59-0.80)]$ in these cohorts (Table 2) (Rothenberg et al., 2010; Kottyan et al., 2014).

For asthma, the best PheWAS results were detected at 17q21 which includes GSDMB and has been previously reported to be associated specifically with childhood onset Asthma (Verlaan et al., 2009). In fact, the best associated SNP rs8067378 in our cohorts $\left[p=3.13 \times 10^{-6}, O R=1.37(1.19-1.57)\right]$, tags the asthma associated haplotype in which the allele-specific expression analyses for this haplotype has previously shown strong association with Asthma risk (Verlaan et al., 2009). There is strong support for this association from a cluster of variants in this neighborhood (Figure 2A).

The minor allele (T) of the intronic SNP rs7903146 in TCF7L2 is one of the larger magnitude and more frequently identified associations in Type 2 diabetes mellitus (T2DM) and hyperlipidemia in many adult GWAS studies (Lyssenko et al., 2007; Huertas-Vazquez et al., 2008). In fact, the best PheWAS trait in our cohorts at this variant was also related to T2DM and hyperlipidemia as well, although our sample size was small. In this family of ICD-9 codes the best suggestive result was obtained for an abnormal glucose test with $[p=0.001, O R=2.00(95 \% \mathrm{CI}$ $1.29-3.08)$ ] (Table 2).

Specifically, for T1DM, in addition to the positive association with PTPN22 mentioned above, additional published loci were confirmed and with relatively larger effect sizes $(O R>2)$ including known HLA-SNP rs660895 $\left[p=7.85 \times 10^{-7}, O R=\right.$ $2.73(95 \% \mathrm{CI}=1.80-4.13)]$, as well as variants near CENPW that previously have been reported for this trait (Table 2) (Barrett et al., 2009).

\section{Other effects}

Several loci previously associated with autism and pervasive developmental disorders (PDD) (GWAS or copy number variations reports) including those at MACROD2, ITGB3, CADM2, and GRIK2 (Jamain et al., 2002; Weiss et al., 2006; Thomas et al., 2008; Anney et al., 2010) also provided evidence of association in our cohorts for these traits (Table 2). Variants in the FOXE1 gene that have been previously associated with primary hypothyroidism and thyroiditis in adult eMERGE cohorts (Denny et al., 2011), produced a trend of association and consistent in 


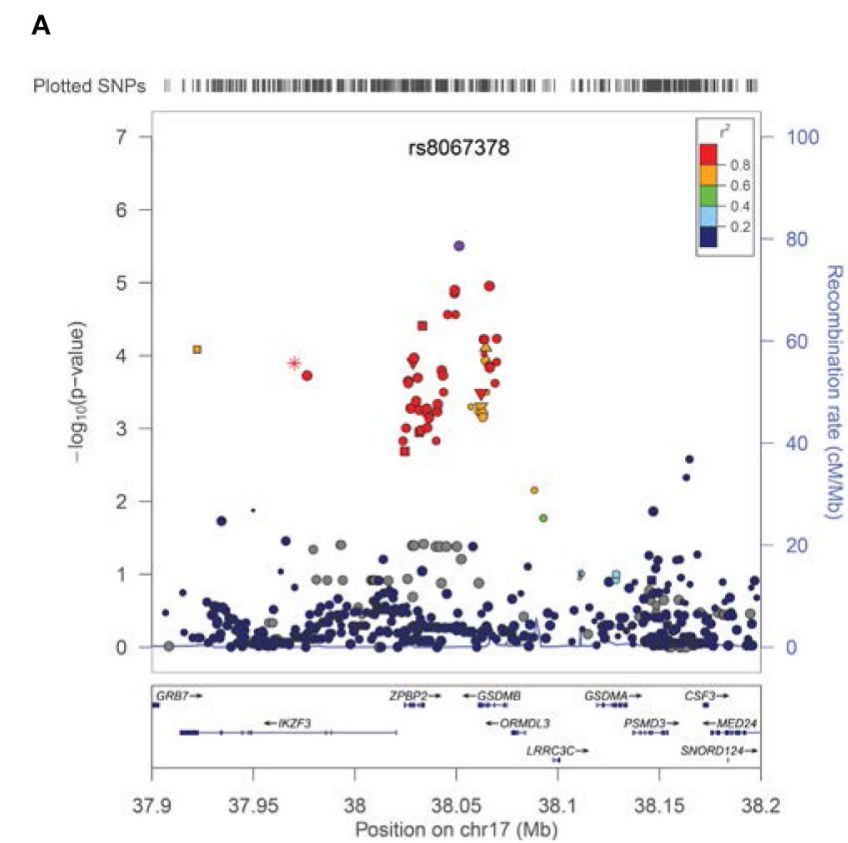

C

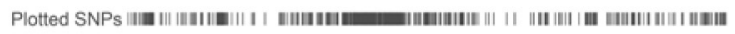

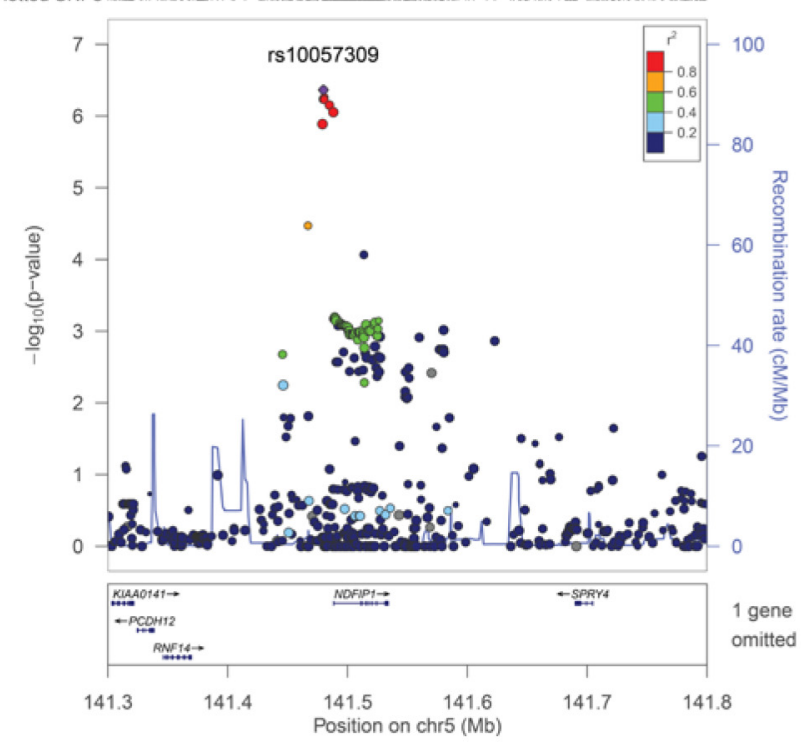

FIGURE 2 | Association results and signals contributing to Asthma, Eosinophilic Esophagitis, Mental Retardation, and Developmental

Delays. SNPs are plotted by position in a $0.2 \mathrm{Mb}$ window against association signals (- $\log 10 P$-value). For each trait, the most significant SNP is highlighted. Estimated recombination rates (from HapMap) are plotted in cyan to reflect the local LD structure. The SNPs surrounding the most significant SNP, are color-coded to reflect their LD with identified SNP (taken
B

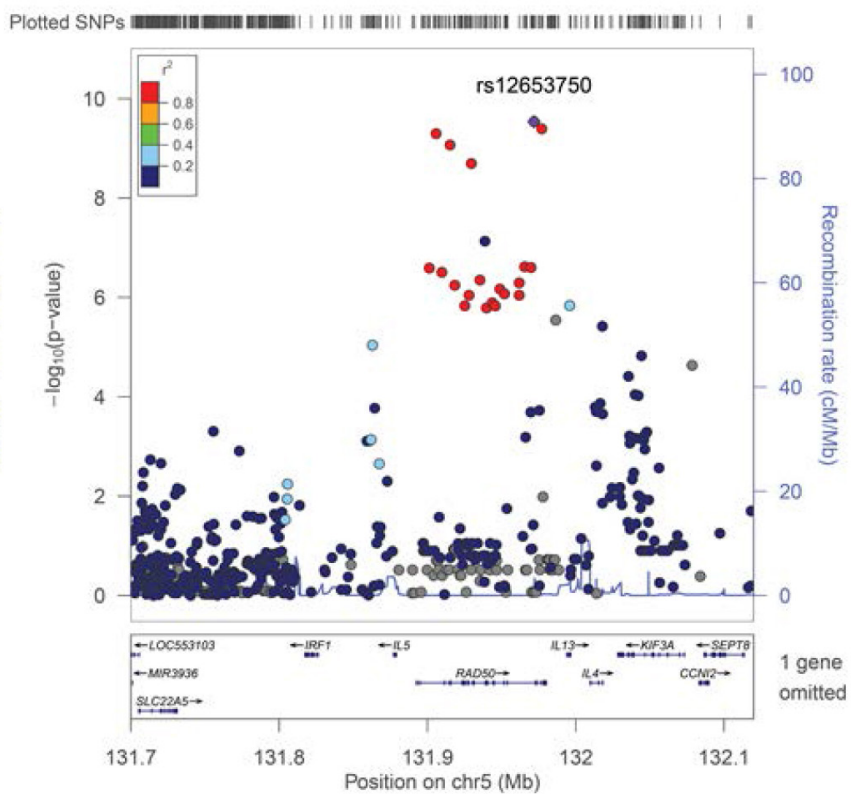

D

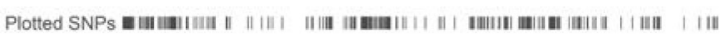

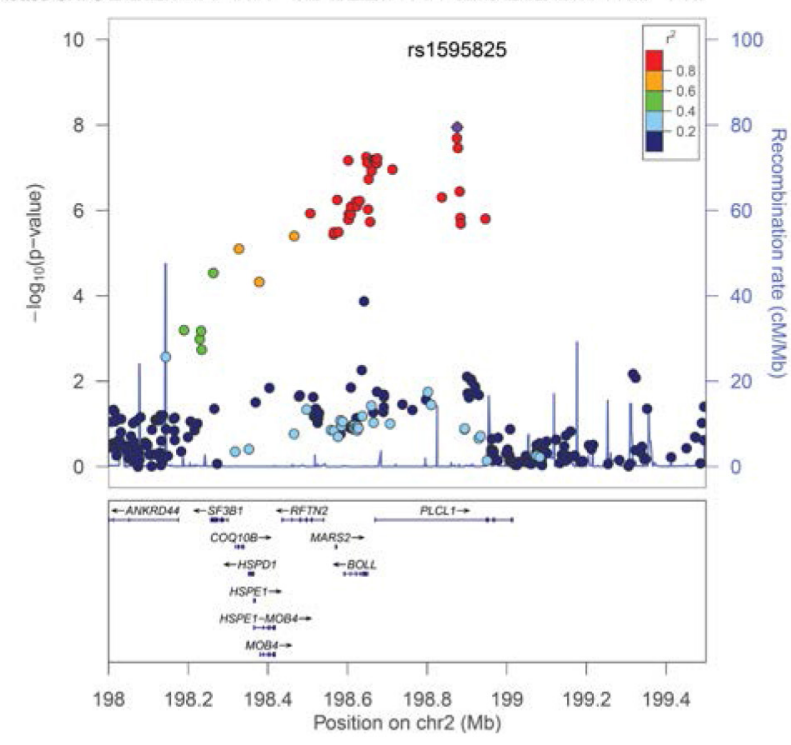

from pairwise $r^{2}$ values from the HapMap CEU database, www.hapmap.org). Regional plots were generated using LocusZoom (http://csg.sph.umich.edu/ locuszoom). (A) Cluster of the association effect for asthma at 17q21 near the gasdermin-B (GSDMB) gene. (B) Association signal for Eosinophilic

Esophagitis at 5q31 (IL5-IL13 cluster region). (C) Cluster of association near the NDFIP1 gene for Mental Retardation traits. (D) Plot of association effects in the PLCL1 region for Developmental Delays-Speech Disorders. directionality with thyroiditis in our pediatric cohorts despite low sample size (Table 2). No gene-gene interaction was evident between PTPN22 and FOXE1 for hypothyroidism in these data. Rs7574865 is a SNP in the third intron of the STAT4 that has been associated with SLE and related autoimmune diseases (Namjou et al., 2009). In these cohorts, pediatric onset lupus was underrepresented (less than 20 cases), however, suggestive associations with wheeze and asthma were detected $[p=0.004$, OR 1.46 $(95 \% \mathrm{CI}=1.11-1.92)$ (Table 2$)]$ with the same direction of the difference in allele frequency previously observed in autoimmune 
traits. This possible association has also been reported in another study (Pykäläinen et al., 2005). Of note, in contrast to rheumatoid arthritis, the STAT4 association effect was weak for JRA in our cohorts (effect size $=1.12, p=0.17$ ). GWAS studies have linked Inflammatory Bowel disease (IBD) to a number of IL-23 pathway genes, in particular IL23R. The well-known coding variant in the IL23 receptor (rs11209026) also showed a trend toward association with IBD in our cohorts with the same allelic direction but due to low sample size (31 cases) it did not reach significance (FDR-q > 0.05) (Li et al., 2010) (data not shown).

\section{Novel findings from this PheWAS}

A number of potentially novel associations remained significant after the permutation procedure to assess the probability of the observed distribution with beta $>0.8$ FDR-q $<0.05$ (Table 3). Variants in the Glucokinase Regulator gene (GCKR) have been previously implicated in metabolic disease, diabetes and hypertriglyceridemia in adults (Bi et al., 2010; Onuma et al., 2010) and were mostly associated with allergic rhinitis in our pediatric cohorts [best SNP rs780093 $p=2.18 \times 10^{-5}, p($ perm $)=8.06 \times$ $\left.10^{-5}, \mathrm{OR}=1.39,95 \% \mathrm{CI}=(1.19-1.61)\right]$ (Table 3), while no significant association was found for diabetes. Indeed, conditional analyses, controlling for diabetes related traits suggest that this is an independent effect $\left(p\right.$-conditional $\left.=6.75 \times 10^{-5}\right)$. Another major regulatory locus for diabetes in adults, JAZF1, also was associated with allergic rhinitis in our cohorts (Table 3) even after controlling for diabetes ( $p$-conditional $=8.46 \times 10^{-5}$, for rs1635852). No significant gene-gene interaction was detected between these two loci or with TCF7L2.

Variants in a cytokine cluster of the IL5-IL13 region, which is known to be associated with Asthma, Allergy, Atopic Dermatitis $(\mathrm{AD})$ and Eosinophilia, produced a cluster of association with EoE in our cohorts [best SNP rs12653750, $p=3.03 \times 10^{-9}$, $P($ perm $\left.)=1.00 \times 10^{-6}, O R=1.73(1.44-2.07)\right]$ (Bottema et al., 2008; Granada et al., 2012). There is a cluster of significant variants in this neighborhood of chromosome 5 (5q31) associated with EoE (Figure 2B). In our cohorts, weaker associations can be detected for all allergy-related phenotypes with the association with Eosinophilia being the most impressive $[p=9.74 \times$ $10^{-5}$ (Table 2)]. However, conditional analyses and controlling for Asthma and Eosinophilia suggest that an independent effect still exists for EoE at this locus using EMR data (conditional $p=9.74 \times 10-5$ for rs20541). Moreover, no long distance linkage disequilibrium between rs 3806932 in TSLP gene at $5 \mathrm{q} 22$ and rs20541 was detected in this population $\left(r^{2}=0.0002, D^{\prime}=0.02\right)$.

We also observed association with $\mathrm{AD}$ within this cytokine cluster consistent with previous reports (Paternoster et al., 2011). However, the best associated SNP for AD (rs272889) was located at SLC22A4 in our population (Table 2). These two variants, rs 272889 and rs12653750, were separated by more than $300 \mathrm{~kb}$ with low linkage disequilibrium $\left(r^{2}<0.1\right)$. A residual effect still exists for $\mathrm{AD}$ and rs 272889 after controlling for EoE status or the rs12653750 variant that suggests a distinct effect $(p$-conditional $=$ $0.002)$. Noteworthy, with regard to $A D$, another reported SNP (rs2897442) downstream of this cluster at KIF3A gene produced only a suggestive association $(p=0.005)$ in our cohort (data not shown).

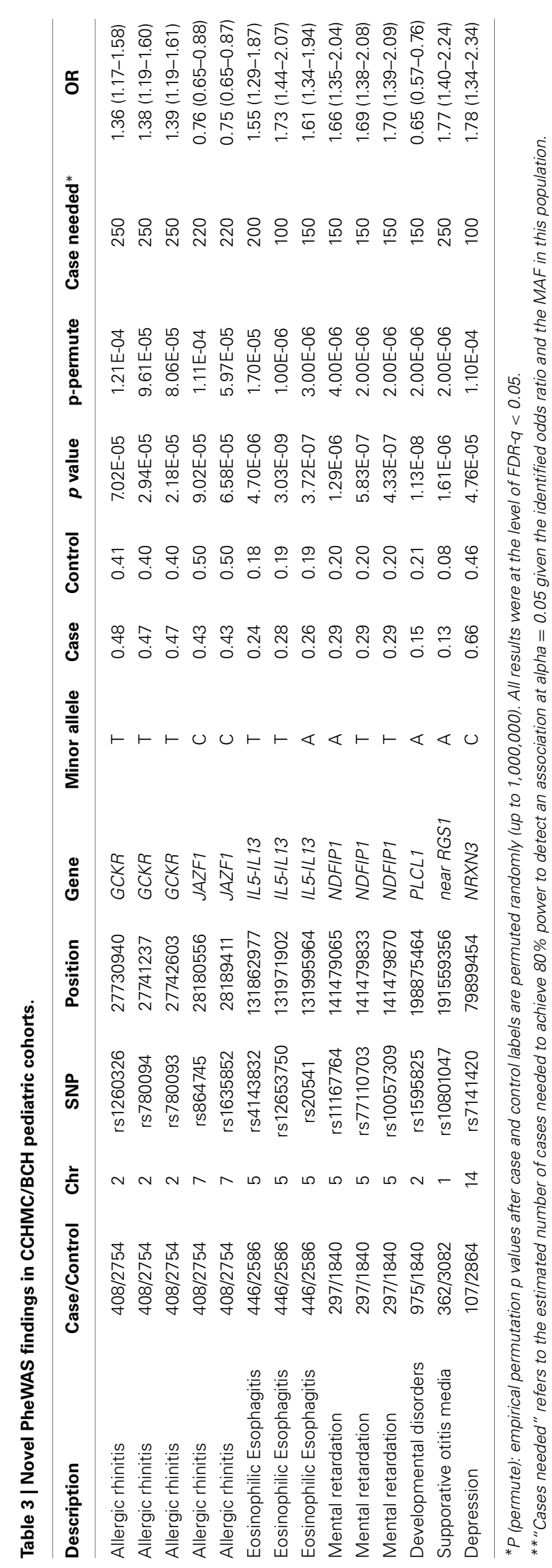


Because of the pleotropic effects between EoE and other allergy related traits, in addition to conditional analyses, we also found possible synergistic effects. One of the closely related phenotypes with EoE is the presence of food allergy. When we combined these two as a subgroup, two additional effects were identified. One cluster was in IL1RL1 that was previously associated with the related phenotype, i.e., allergy and asthma (best SNP rs3771180, $p=5.71 \times 10^{-5}$, Table 2, Torgerson et al., 2011) and another was in CLEC16A, previously associated with different autoimmune diseases [best SNP rs12924729, $p=3.34 \times 10^{-8}$ (Table 2), (Mells et al., 2011)] and was reported as a suggestive effect in recent GWAS study for EoE (Kottyan et al., 2014).

Variants near RGS cluster of genes on chromosome 1, previously reported to be associated with IBD and other autoimmune diseases (Hunt et al., 2008; Esposito et al., 2010), were associated with susceptibility to infection, in particular suppurative otitis media [best SNP rs10801047, $p=1.61 \times 10^{-6}$, $P$ (perm) $\left.=2.00 \times 10^{-6}, O R=1.7795 \% \mathrm{CI}=1.398-2.24\right]$.

New association signals have been detected near the NDFIP1 gene for mental retardation related traits. Variants near this gene that is expressed mostly in brain, were previously reported to be associated with IBD through an unknown mechanism and with a risk effect for major allele (SNP $=$ rs11167764) (Franke et al., 2010). Instead, we found a risk effect for the minor allele [best SNP rs10057309, $p=4.33 \times 10^{-7}, p($ perm $)=2.00 \times 10^{-6}, O R=$ 1.702 , $95 \%$ CI $=1.38-2.09$ ] (Table 3). Similarly, cerebral palsy, which is linked to mental retardation, was also associated with this variant $\left(p=9.00 \times 10^{-4}\right)$. However, conditional analyses controlling for cerebral palsy suggest an independent effect for overall mental retardation (conditional $p=8.00 \times 10^{-4}$ ). Furthermore, excluding the small number of samples with known chromosomal abnormalities $(N<40)$ did not affect this result. The overall cluster effect in this neighborhood for mental retardation bolsters the suspicion that an association is found here (Figure 2C).

Additionally, for developmental delays of speech and language, a novel signal effect was detected in the PLCL1 gene at chromosome 2 [best SNP rs1595825, $p=1.13 \times 10^{-8}, O R=0.65$ $(0.57-0.76)]$ (Figure 2D, Table 3). Weaker associations $(0.01>$ $p>0.00001)$ were also detected for related neurologic phenotypes including abnormal movement, lack of coordination and epilepsy at this locus (data not shown).

NRXN3 polymorphisms that have been previously reported to be associated with substance dependence (Docampo et al., 2012), smoking behavior and attention related problems (Stoltenberg et al., 2011), were associated with depression in our pediatric cohorts (Table 3 Noteworthy, the major allele of our reported SNP (rs7141420) has been linked to obesity in adult cohorts (Berndt et al., 2013), while we found association with the minor allele for depression $\left[p=4.76 \times 10^{-5}, O R=1.78(1.34-2.34)\right.$, Table 3]. Furthermore, rare micro-deletions in this gene were previously reported for Autism case reports but these rare variants are not available to assess in our genotyped cohorts (Vaags et al., 2012).

\section{DISCUSSION}

This first pediatric PheWAS finds 38 associations, 24 previously known phenotype-genotype associations in a pediatric population using EMR-linked eMERGE databases and identified 14 new possible associations at beta $>0.8$ and FDR-q $<$ 0.05 . From analysis performed on EMR-linked data from 4268 European individuals, we successfully confirmed several major effects for phenotypes with moderate to large sample size, in particular for Asthma, Autism, and neurodevelopmental disease as well as several effects for Type 1 and Type 2 Diabetes (T1DM, T2DM) and Thyroiditis. Almost all of the significant phenotype associations were with common variants ( $\mathrm{MAF}>10 \%$ ) (Tables 2, 3). In addition, we compared and verified the consistency of allele frequency of reported markers among cohorts, sample collection sites and with CEU-Hapmap data. Considering a desired power of 0.8 , for variants at the fixed allele frequency of $10 \%$ and size effect of 1.5 or above, 200 cases are sufficient to detect association at an alpha level of 0.05 . Indeed, we have surpassed this level for most of our reported traits. In addition, for all reported phenotypes the control sample was at least two or three times larger than cases (Tables 2, 3). Importantly, since our control samples for each trait are an EMR-derived population and not healthy individuals, this large number of control samples provides minor allele frequencies consistent with hapmap-CEU frequencies for all of our reported variants.

The results for JRA and EoE depend upon previously published studies of these phenotypes. While the case samples are mostly identical, the control samples were substantially different. Consequently, we cannot refer to these particular findings as constituting confirmation and yet our results and different methodology support the previous reports.

In addition, we also identified several novel PheWAS findings for pediatric traits in particular for Allergic Rhinitis, Otitis Media, EoE, Mental Retardation, and Developmental Delays all with sufficient power (beta $>0.8$ ) (Table 3, Figures 2B-D). This study, however, is underpowered to make discoveries for rare variants or uncommon traits. The power to detect a finding in PheWAS is determined by many factors, including sample size, risk allele frequency, effect size, model of inheritance, the effect of environment and the prevalence of a phenotype within the population.

Similar to previous studies, we also observed pleiotropy for a number of loci in particular PTPN22 for JRA, T1DM, and Thyroiditis, IL5 for Eosinophilia, Asthma, and EoE and NDFIP1 for Mental Retardation traits and Cerebral Palsy. These pleotropic effects are specifically expected to be due to underlying biologic correlations. On the other hand, we rarely observed simultaneous robust associations with multiple unrelated phenotypes that had sufficient power. Furthermore, one of the advantages of PheWAS studies is the ability to control the granularity of a database with regard to related phenotypes. For example, by combining two related phenotypes such as uveitis with JRA or food allergy with EoE, we were able to evaluate new subgroups and identify new loci responsible for shared underlying pathways that otherwise cannot be detected or require much larger sample sizes. Further studies with larger sample sizes would be useful to test and perhaps corroborate these findings.

Association of Allergic Rhinitis with loci responsible for diabetes in adults (GCKR-JAZF1) may highlight a shared underlying mechanism. In fact, the connection between allergy and diabetes 
has been previously suggested in humans but cannot be explained by the Th1/Th2 paradigm (Dales et al., 2005). Moreover, in animal experiments, treating mice with mast cell-stabilizing agents reduced diabetes manifestations (Liu et al., 2009). It is also possible that in our pediatric cohorts we have under-diagnosed children who are diagnosed with diabetes which would appear in a later stage of development. In fact, GCKR is an inhibitor of glucokinase $(G C K)$, a gene responsible for the autosomal dominant form of T2DM that usually develops later in life and in adulthood. Of note, neither of these two loci showed significant association with Body Mass Index (BMI) in our previous report with these data nor has the obesity link been established in adult studies (Namjou et al., 2013).

The novel association of a cytokine cluster in the IL5-IL13 region for the EoE trait is particularly interesting since antiIL5 monoclonal antibodies have been recommended as a novel therapeutic agent for EoE and other eosinophilia-related traits (Corren, 2012). In general, both IL5 and IL13 play a major role for regulation of maturation, recruitment, and survival of eosinophils and the variant reported here has been previously associated with other allergic-related traits and with the same direction of allele frequency difference (Bottema et al., 2008; Granada et al., 2012). In particular, a non-synonymous polymorphism in the IL13 gene, rs20541 (R130Q) (Table 3), has been shown to be associated with increased IL-13 protein activity, altered IL-13 production, and increased binding of nuclear proteins to this region (van der Pouw Kraan et al., 1999). Perhaps, the association is a reflection of linkage disequilibrium with another polymorphism in the $5 \mathrm{q} 31$ region. In fact, in our analyses residual effect still exists for the best SNP (rs12653750), shown in Figure 2B after controlling for rs20541 ( $p$-conditional = $\left.2.27 \times 10^{-5}\right)\left(r^{2}=0.35\right)$. This possible association did not reach significance in previous GWAS studies for EoE and had only produced a suggestive effect $(0.05<p<0.001)$. Perhaps, this behavior is explained partly by phenotypic heterogeneity since minor allele frequency of independent set of both control populations were the same. Indeed, we found that those with the subphenotype of EoE with Eosinophilia had the strongest size effect $(O R=1.83,95 \% \mathrm{CI}=1.44-2.32)$ and our cohorts were enriched with this subphenotype [177 of total $446 \mathrm{EoE}$ cases $(40 \%)]$. Of note, the SNPs in this region were originally selected because of eosinophilia-related publications (Bottema et al., 2008; Granada et al., 2012).

Moreover, combining subgroups of patients with food allergy and EoE revealed two new loci that may explain shared etiology. Indeed, the connection between allergy and Interleukin 1 receptor-like-1 (IL1R1) is already known (Torgerson et al., 2011). The ligand for IL1R1, IL-33, is a potent eosinophil activator (Bouffi et al., 2013). Interestingly, there is also a report of association of CLEC16A variants with allergy in large analysis with more than 50,000 subjects from 23andMe Inc. (Hinds et al., 2013). Ctype lectin domain family 16 , also known as CLEC16A, is mostly associated with autoimmune related traits and is highly expressed in B lymphocytes and natural killer cells. The molecular and cellular functions of CLEC16A are currently under investigation.

Our conditional analyses suggest an independent effect at the SLC22A4 gene for Atopic Dermatitis. This solute carrier family gene is predominantly expressed in CD14 cells and has an important role for elimination of many endogenous small organic cations as well as a wide array of drugs and environmental toxins. The associated SNP, rs272889, has been previously shown to be correlated with blood metabolite concentration (Suhre et al., 2011). Other variants in this gene were associated with IBD and Crohns disease as well (Feng et al., 2009). Of note, a key substrate of this transporter is ergothioneine, a natural antioxidant, which Mammalia acquire exclusively from their food. Ergothionine is a powerful antioxidant though its precise physiological purpose remains unclear.

Asthma is associated at the 17q21 in our cohorts (Figure 1). The best associated SNP, rs8067378, is known to function as a cisregulatory variant that correlates with expression of the GSDMB gene (Verlaan et al., 2009). Variants in GSDMB have been shown to determine multiple asthma related phenotypes specifically in childhood asthma including associations with lung function and disease severity (Tulah et al., 2013). These gasdermin-family genes are implicated in the regulation of apoptosis mostly in epithelial cells and have also been linked to cancer; however, their actual function with respect to disease association remains unknown. The associated variants in this cluster are suspected to be regulatory SNPs that govern the transcriptional activity of at least three nearby genes (ZPBP2, GSDMB, and ORMDL3) (Verlaan et al., 2009).

We confirmed several loci responsible for Autism and Pervasive Developmental Disease including MACROD2, ITGB3, CADM2, and GRIK2. ITGB3 has been known as a quantitative trait locus (QTL) for whole blood serotonin levels (Weiss et al., 2004, 2006). Serotonin is a monoamine neurotransmitter that has long been implicated in the etiology of Autism. In fact, about 30 percent of patients with autism have abnormal blood serotonin levels (Weiss et al., 2004). Similarly, GRIK2 is an ionotropic glutamate receptor associated with autism (Cook, 1990; Cook et al., 1997). CADM2 is a member of the synaptic cell adhesion molecule with roles in early postnatal development of the central nervous system (Thomas et al., 2008). The function of MACROD2 (previously c20orf133) is still largely unknown. For Autism that is more commonly seen in males, we found no significant gender effect for these loci.

Association of variants in the neighborhood of RGS cluster genes with suppurative otitis media is another novel finding. SNPs in this region have been previously linked to celiac disease, multiple sclerosis and other autoimmune diseases (Hunt et al., 2008; Esposito et al., 2010). The link between susceptibility to infection and autoimmunity has been long suggested given the fact that the level and regulation of RGS proteins in lymphocytes also significantly impact lymphocyte migration and function. In our pediatric cohort the number of patients with celiac disease was small $(n=23)$ and the association was not detected. Interestingly, one of the major risk variant for celiac disease, rs13151961 (KIAA1109), as well as known HLA variants, produced a tread toward association for celiac disease but did not pass the FDR threshold (data not shown).

Finally we also detected a novel association between mental retardation and the NDFIP1 gene (Figure 2C, Table 3). Of note, no effect was detected with Autism at this locus. Indeed, the 
only other effect observed in this region was related to Cerebral Palsy $\left(p=9.00 \times 10^{-4}\right)$ and, as mentioned above, an independent effect exists for Mental Retardation. The PheWAS code for mental retardation includes ICD-9 codes for mild, moderate and profound degrees of retardation as well as not-otherwisespecified (MR-NOS). Indeed, an additive correlation can also be detected when we score these subgroups according to severity excluding the MR-NOS subgroup $\left(p=3.00 \times 10^{-4}\right)$. Larger sample size is necessary to fully elucidate this interesting effect. The Nedd4 family-interacting protein 1 (Ndfip1) is an adaptor protein for the Nedd 4 family of E3 ubiquitin ligases important for axon and dendrite development. In fact, cerebral atrophy is one of the main findings in Ndfip1 KO mice (Hammond et al., 2014). Another neurodevelopmental association effect was observed in the vicinity of the Phospholipase C-Like 1 (PLCL1, PRIP-1) gene for overall Developmental Delays-Speech and Language Disorder (Table 3, Figure 2D). This gene which is expressed predominantly in brain, regulates the turnover of GABA-receptors, contributes to the maintenance of GABA-mediated synaptic inhibition, and has been implicated in several pathologies in animal models and human including epilepsy, bone density and cancer (Liu et al., 2008; Zhu et al., 2012). Finally, we also detected a link between Neuroxin-3 and early onset depression in this study (Table 3 ). In fact, this gene has a major role in synaptic plasticity and function in the nervous system as a receptor and cell adhesion molecule.

In summary, by using the PheWAS approach and re-mapping the ICD-9 codes on our European ancestry pediatric cohorts we have been able to verify and confirm a variety of previously reported associations as well as discover new effects that potentially have clinical implications. Similar to adult PheWAS studies, our data also support the importance of this approach in pediatrics. We replicated known phenotype-genotype associations in a pediatric population using these EMR-linked eMERGE databases, and also noted a number of new possible associations that warrant additional study, especially including the relationship of PLCL1 to speech and language development and IL5-IL13 to EoE. Some of the limitations to the current PheWAS map include the fact that current map does not take into account of the correlation between some phenotypes and treat them as independent. Future pediatric PheWAS directions will include enhancements of a PheWAS map for more precise modeling of trait associations as well as improvements for richer querying and filtering.

\section{ACKNOWLEDGMENTS}

We are grateful to the individuals who participated in this study. We thank the genotyping core facilities in both academic centers $(\mathrm{CCHMC}, \mathrm{BCH})$ and our colleagues who facilitated the genotyping and recruitment of subjects.

This work was supported by a grant from the National Human Genomic Research Institute: 1U01HG006828 with other NIH support (R37 AI024717, P01 AI083194, U19 AI066738, and P01 AR049084), the US Department of Veterans Affairs, the Campaign Urging Research For Eosinophilic Diseases (CURED) Foundation, as well as the Food Allergy Research Education (FARE) Foundation.

\section{REFERENCES}

Anney, R., Klei, L., Pinto, D., Regan, R., Conroy, J., Magalhaes, T. R., Correia, C., et al. (2010). A genome-wide scan for common alleles affecting risk for autism. Hum. Mol. Genet. 19, 4072-4082. doi: 10.1093/hmg/ddq307

Barrett, J. C., Clayton, D. G., Concannon, P., Akolkar, B., Cooper, J. D., Erlich, H. A., et al. (2009). Genome-wide association study and meta-analysis find that over 40 loci affect risk of type 1 diabetes. Nat. Genet. 41, 703-707. doi: 10.1038/ng.381

Berndt, S. I., Gustafsson, S., Mägi, R., Ganna, A., Wheeler, E., Feitosa, M. F., et al. (2013). Genome-wide meta-analysis identifies 11 new loci for anthropometric traits and provides insights into genetic architecture. Nat. Genet. 45, 501-512. doi: 10.1038/ng.2606

Bi, M., Kao, W. H., Boerwinkle, E., Hoogeveen, R. C., Rasmussen-Torvik, L. J., Astor, B. C., et al. (2010). Association of rs780094 in GCKR with metabolic traits and incident diabetes and cardiovascular disease: the ARIC Study. PLoS ONE 5:e11690. doi: 10.1371/journal.pone.0011690

Bottema, R. W., Reijmerink, N. E., Kerkhof, M., Koppelman, G. H., Stelma, F. F., Gerritsen, J., et al. (2008). Interleukin 13, CD14, pet and tobacco smoke influence atopy in three Dutch cohorts: the allergenic study. Eur. Respir. J. 32, 593-602. doi: 10.1183/09031936.00162407

Bouffi C. 1st., Rochman, M., Zust, C. B., Stucke, E. M., Kartashov, A., Fulkerson, P. C., et al. (2013). IL-33 markedly activates murine eosinophils by an NF-?Bdependent mechanism differentially dependent upon an IL-4-driven autoinflammatory loop. J. Immunol. 91, 4317-4325. doi: 10.4049/jimmunol.1301465

Carroll, R. J., Bastarache, L., and Denny, J. C. (2014). R PheWAS: data analysis and plotting tools for phenome-wide association studies in the R environment. Bioinformatics 30, 2375-2376. doi: 10.1093/bioinformatics/btu197

Cook, E. H. Jr., Courchesne, R., Lord, C., Cox, N. J., Yan, S., Lincoln, A., et al. (1997). Evidence of linkage between the serotonin transporter and autistic disorder. Mol. Psychiatry 2, 247-250.

Cook, E. H. (1990). Autism: review of neurochemical investigation. Synapse 6, 292-308. doi: 10.1002/syn.890060309

Corren, J. (2012). Inhibition of interleukin-5 for the treatment of eosinophilic diseases. Discov. Med. 13, 305-312.

Dales, R., Chen, Y., Lin, M., and Karsh, J. (2005). The association between allergy and diabetes in the Canadian population: implications for the Th1-Th2 hypothesis. Eur. J. Epidemiol. 20, 713-717. doi: 10.1007/s10654-005-7920-1

Denny, J. C., Bastarache, L., Ritchie, M. D., Carroll, R. J., Zink, R., Mosley, J. D., et al. (2013). Systematic comparison of phenome-wide association study of electronic medical record data and genome-wide association study data. Nat. Biotechnol. 31, 1102-1110. doi: 10.1038/nbt.2749

Denny, J. C., Crawford, D. C., Ritchie, M. D., Bielinski, S. J., Basford, M. A., Bradford, Y., et al. (2011). Variants near FOXE1 are associated with hypothyroidism and other thyroid conditions: using electronic medical records for genome- and phenome-wide studies. Am. J. Hum. Genet. 89, 529-542. doi: 10.1016/j.ajhg.2011.09.008

Denny, J. C., Ritchie, M. D., Basford, M. A., Pulley, J. M., Bastarache, L., BrownGentry, K., et al. (2010). PheWAS: demonstrating the feasibility of a phenomewide scan to discover gene-disease associations. Bioinformatics 26, 1205-1210. doi: 10.1093/bioinformatics/btq126

Docampo, E., Ribasés, M., Gratacòs, M., Bruguera, E., Cabezas, C., SánchezMora, C., et al. (2012). Association of Neurexin 3 polymorphisms with smoking behavior. Genes Brain Behav. 11, 704-711. doi: 10.1111/j.1601183X.2012.00815.x

Esposito, F., Patsopoulos, N. A., Cepok, S., Kockum, I., Leppä, V., Booth, D. R., et al. (2010). IL12A, MPHOSPH9/CDK2AP1 and RGS1 are novel multiple sclerosis susceptibility loci. Genes Immun. 11, 397-405. doi: 10.1038/gene.2010.28

Feng, Y., Zheng, P., Zhao, H., and Wu, K. (2009). SLC22A4 and SLC22A5 gene polymorphisms and Crohn's disease in the Chinese Han population. J. Dig. Dis. 10, 181-187. doi: 10.1111/j.1751-2980.2009.00383.x

Franke, A., McGovern, D. P., Barrett, J. C., Wang, K., Radford-Smith, G. L., Ahmad, T., et al. (2010). Genome-wide meta-analysis increases to 71 the number of confirmed Crohn's disease susceptibility loci. Nat. Genet. 12, 1118-1125. doi: 10.1038/ng.717

Gauderman, W. J., and Morrison, J. M. (2006). QUANTO 1.1: A Computer Program for Power and Sample Size Calculations for Genetic-epidemiology Studies. Available online at: http://hydra.usc.edu/gxe

Gidding, S. S. (1993). The rationale for lowering serum cholesterol levels in American children. Am. J. Dis. Child. 147, 386-392. 
Granada, M., Wilk, J. B., Tuzova, M., Strachan, D. P., Weidinger, S., Albrecht, E., et al. (2012). A genome-wide association study of plasma total IgE concentrations in the Framingham Heart Study. J. Allergy Clin. Immunol. 129, 840-845.e21. doi: 10.1016/j.jaci.2011.09.029

Hammond, V. E., Gunnersen, J. M., Goh, C. P., Low, L. H., Hyakumura, T., Tang, M. M., et al. (2014). Ndfipl is required for the development of pyramidal neuron dendrites and spines in the neocortex. Cereb. Cortex 24, 3289-3300. doi: $10.1093 /$ cercor/bht191

Hinds, D. A., McMahon, G., Kiefer, A. K., Do, C. B., Eriksson, N., Evans, D. M., et al. (2013). A genome-wide association meta-analysis of self-reported allergy identifies shared and allergy-specific susceptibility loci. Nat. Genet. 45, 907-911. doi: 10.1038/ng.2686

Hinks, A., Cobb, J., Marion, M. C., Prahalad, S., Sudman, M., Bowes, J., et al. (2013). Dense genotyping of immune-related disease regions identifies 14 new susceptibility loci for juvenile idiopathic arthritis. Nat. Genet. 45, 664-669. doi: 10.1038/ng.2614

Howie, B., Marchini, J., and Stephens, M. (2011). Genotype imputation with thousands of genomes. G3 (Bethesda). 1, 457-470. doi: 10.1534/g3.111. 001198

Huertas-Vazquez, A., Plaisier, C., Weissglas-Volkov, D., Sinsheimer, J., CanizalesQuinteros, S., Cruz-Bautista, I., et al. (2008). TCF7L2 is associated with high serum triacylglycerol and differentially expressed in adipose tissue in families with familial combined hyperlipidaemia. Diabetologia 51, 62-69. doi: 10.1007/s00125-007-0850-6

Hunt, K. A., Zhernakova, A., Turner, G., Heap, G. A., Franke, L., Bruinenberg, M., et al. (2008). Newly identified genetic risk variants for celiac disease related to the immune response. Nat. Genet. 40, 395-402. doi: 10.1038/ng.102

Jamain, S., Betancur, C., Quach, H., Philippe, A., Fellous, M., Giros, B., et al. (2002). Linkage and association of the glutamate receptor 6 gene with autism. Mol. Psychiatry 7, 302-310. doi: 10.1038/sj.mp.4000979

Klein, J. D., Sesselberg, T. S., Johnson, M. S., O’Connor, K. G., Cook, S., Coon, M., et al. (2010). Adoption of body mass index guidelines for screening and counseling in pediatric practice. Pediatrics 125, 265-272. doi: 10.1542/peds.2008-2985

Kottyan, L. C., Davis, B., Sherrill, J. D., Liu, K., Rochman, M., Kaufman, K., et al. (2014). Identification of genome-wide susceptibility loci for eosinophilic esophagitis elucidates tissue-specificity of this allergic disease. Nat. Genet. 46, 895-900. doi: 10.1038/ng.3033

Lee, H. S., Kang, J., Yang, S., Kim, D., and Park, Y. (2011). Susceptibility influence of a PTPN22 haplotype with thyroid autoimmunity in Koreans. Diabetes Metab. Res. Rev. 27, 878-882. doi: 10.1002/dmrr.1265

Li, Y., Mao, Q., Shen, L., Tian, Y., Yu, C., Zhu, W. M., et al. (2010). Interleukin23 receptor genetic polymorphisms and Crohn's disease susceptibility: a metaanalysis. Inflamm. Res. 59, 607-614. doi: 10.1007/s00011-010-0171-y

Liao, K. P., Diogo, D., Cui, J., Cai, T., Okada, Y., Gainer, V. S., et al. (2014). Association between low density lipoprotein and rheumatoid arthritis genetic factors with low density lipoprotein levels in rheumatoid arthritis and non-rheumatoid arthritis controls. Ann. Rheum. Dis. 73, 1170-1175. doi: 10.1136/annrheumdis-2012-203202

Liu, J., Divoux, A., Sun, J., Zhang, J., Clément, K., Glickman, J. N., Sukhova, G. K., et al. (2009). Genetic deficiency and pharmacological stabilization of mast cells reduce diet-induced obesity and diabetes in mice. Nat. Med. 15, 940-945. doi: 10.1038/nm.1994

Liu, Y. Z., Wilson, S. G., Wang, L., Liu, X. G., Guo, Y. F., Li, J., et al. (2008). Identification of PLCL1 gene for hip bone size variation in females in a genomewide association study. PLoS ONE 3:e3160. doi: 10.1371/journal.pone.0003160

Lyssenko, V., Lupi, R., Marchetti, P., Del Guerra, S., Orho-Melander, M., Almgren, P., et al. (2007). Mechanisms by which common variants in the TCF7L2 gene increase risk of type 2 diabetes. J. Clin. Invest. 117, 2155-2163. doi: 10.1172/JCI30706

Mells, G. F., Floyd, J. A., Morley, K. I., Cordell, H. J., Franklin, C. S., Shin, S. Y., et al. (2011). Genome-wide association study identifies 12 new susceptibility loci for primary biliary cirrhosis. Nat. Genet. 43, 329-332. doi: 10.1038/ng.789

Namjou, B., Keddache, M., Marsolo, K., Wagner, M., Lingren, T., Cobb, B., et al. (2013). EMR-linked GWAS study: investigation of variation landscape of loci for body mass index in children. Front. Genet. 4:268. doi: 10.3389/fgene.2013.00268

Namjou, B., Sestak, A. L., Armstrong, D. L., Zidovetzki, R., Kelly, J. A., Jacob, N., et al. (2009). High-density genotyping of STAT4 reveals multiple haplotypic associations with systemic lupus erythematosus in different racial groups. Arthritis Rheum. 60, 1085-1095. doi: 10.1002/art.24387
Neuraz, A., Chouchana, L., Malamut, G., Le Beller, C., Roche, D., Beaune, P., et al. (2013). Phenome-wide association studies on a quantitative trait: application to TPMT enzyme activity and thiopurine therapy in pharmacogenomics. PLoS Comput. Biol. 9:e1003405. doi: 10.1371/journal.pcbi.1003405

Onuma, H., Tabara, Y., Kawamoto, R., Shimizu, I., Kawamura, R., Takata, Y., et al. (2010). The GCKR rs780094 polymorphism is associated with susceptibility of type 2 diabetes, reduced fasting plasma glucose levels, increased triglycerides levels and lower HOMA-IR in Japanese population. J. Hum. Genet. 55, 600-604. doi: 10.1038/jhg.2010.75

Paternoster, L., Standl, M., Chen, C. M., Ramasamy, A., Bønnelykke, K., Duijts, L., et al. (2011). Meta-analysis of genome-wide association studies identifies three new risk loci for atopic dermatitis. Nat. Genet. 44, 187-192. doi: 10.1038/ng.1017

Pendergrass, S. A., Brown-Gentry, K., Dudek, S., Frase, A., Torstenson, E. S., Goodloe, R., et al. (2013). Phenome-wide association study (PheWAS) for detection of pleiotropy within the Population Architecture using Genomics and Epidemiology (PAGE) Network. PLoS Genet. 9:e1003087. doi: 10.1371/journal.pgen.1003087

Pendergrass, S. A., Brown-Gentry, K., Dudek, S. M., Torstenson, E. S., Ambite, J. L., Avery, C. L., et al. (2011). The use of phenome-wide association studies (PheWAS) for exploration of novel genotype-phenotype relationships and pleiotropy discovery. Genet Epidemiol. 35, 410-422. doi: 10.1002/gepi.20589

Plenge, R. M., Seielstad, M., Padyukov, L., Lee, A. T., Remmers, E. F., Ding, B., et al. (2007). TRAF1-C5 as a risk locus for rheumatoid arthritis-a genomewide study. N. Engl. J. Med. 357, 1199-1209. doi: 10.1056/NEJMoa073491

Price, A. L., Patterson, N. J., Plenge, R. M., Weinblatt, M. E., Shadick, N. A., and Reich, D. (2006). Principal components analysis corrects for stratification in genome-wide association studies. Nat. Genet. 38, 904-909. doi: 10.1038/ng1847

Pruim, R. J., Welch, R. P., Sanna, S., Teslovich, T. M., Chines, P. S., Gliedt, T. P., et al. (2010). LocusZoom: regional visualization of genome-wide association scan results. Bioinformatics 26, 2336-2337. doi: 10.1093/bioinformatics/btq419

Purcell, S., Neale, B., Todd-Brown, K., Thomas, L., Ferreira, M. A., Bender, D., et al. (2007). PLINK: a tool set for whole-genome association and populationbased linkage analyses. Am. J. Hum. Genet. 81, 559-575. doi: 10.1086/ 519795

Pykäläinen, M., Kinos, R., Valkonen, S., Rydman, P., Kilpeläinen, M., Laitinen, L. A., et al. (2005). Association analysis of common variants of STAT6, GATA3, and STAT4 to asthma and high serum IgE phenotypes. J. Allergy Clin. Immunol. 115, 80-87. doi: 10.1016/j.jaci.2004.10.006

Ritchie, M. D., Denny, J. C., Zuvich, R. L., Crawford, D. C., Schildcrout, J. S., Bastarache, L., et al. (2013). Genome-and phenome-wide analyses of cardiac conduction identifies markers of arrhythmia risk. Circulation 127, 1377-1385. doi: 10.1161/CIRCULATIONAHA.112.000604

Rothenberg, M. E., Spergel, J. M., Sherrill, J. D., Annaiah, K., Martin, L. J., Cianferoni, A., et al. (2010). Common variants at $5 \mathrm{q} 22$ associate with pediatric eosinophilic esophagitis. Nat. Genet. 42, 289-291. doi: 10.1038/ng.547

Setia, S., Andrade, M., Tromp, G., Kuivaniemi, H., Pugh, E., Namjou, B., et al. (2014). Imputation and quality control steps for combining multiple genomewide datasets. Front. Genet. 5:370. doi: 10.3389/fgene.2014.00370

Stoltenberg, S. F., Lehmann, M. K., Christ, C. C., Hersrud, S. L., and Davies, G. E. (2011). Associations among types of impulsivity, substance use problems and neurexin-3 polymorphisms. Drug Alcohol Depend. 119, e31-e38. doi: 10.1016/j.drugalcdep.2011.05.025

St Pourcain, B., Skuse, D. H., Mandy, W. P., Wang, K., Hakonarson, H., Timpson, N. J., et al. (2014). Variability in the common genetic architecture of socialcommunication spectrum phenotypes during childhood and adolescence. Mol. Autism 5:18. doi: 10.1186/2040-2392-5-18

Suhre, K., Shin, S. Y., Petersen, A. K., Mohney, R. P., Meredith, D., Wägele, B., et al. (2011). Human metabolic individuality in biomedical and pharmaceutical research. Nature 477, 54-60. doi: 10.1038/nature10354

Thomas, L. A., Akins, M. R., and Biederer, T. (2008). Expression and adhesion profiles of SynCAM molecules indicate distinct neuronal functions. J. Comp. Neurol. 510, 47-67. doi: 10.1002/cne.21773

Thompson, S. D., Marion, M. C., Sudman, M., Ryan, M., Tsoras, M., Howard, T. D., et al. (2012). Genome-wide association analysis of juvenile idiopathic arthritis identifies a new susceptibility locus at chromosomal region 3q13. Arthritis Rheum. 64, 2781-2791. doi: 10.1002/art.34429

Tiisala, R., and Kantero, R. L. (1971). Studies on growth of Finnish children from birth to 10 years. 3. Comparison of height and weight distance curves based 
on longitudinal and cross-sectional series from birth to 10 years. Acta Paediatr Scand. Suppl. 220, 13-7.

Todd, J. A., Walker, N. M., Cooper, J. D., Smyth, D. J., Downes, K., Plagnol, V., et al. (2007). Robust associations of four new chromosome regions from genomewide analyses of type 1 diabetes. Nat. Genet. 39, 857-864. doi: 10.1038/ng2068

Torgerson, D. G., Ampleford, E. J., Chiu, G. Y., Gauderman, W. J., Gignoux, C. R., Graves, P. E., et al. (2011). Meta-analysis of genome-wide association studies of asthma in ethnically diverse North American populations. Nat. Genet. 43, 887-892. doi: $10.1038 /$ ng. 888

Tulah, A. S., Holloway, J. W., and Sayers, I. (2013). Defining the contribution of SNPs identified in asthma GWAS to clinical variables in asthmatic children. BMC Med. Genet. 14:100. doi: 10.1186/1471-2350-14-100

Vaags, A. K., Lionel, A. C., Sato, D., Goodenberger, M., Stein, Q. P., Curran, S., et al. (2012). Rare deletions at the neurexin 3 locus in autism spectrum disorder. Am. J. Hum. Genet. 90, 133-141. doi: 10.1016/j.ajhg.2011.11.025

van der Pouw Kraan, T. C., van Veen, A., Boeije, L. C., van Tuyl, S. A., de Groot, E. R., Stapel, S. O., et al. (1999). An IL-13 promoter polymorphism associated with increased risk of allergic asthma. Genes Immun. 1, 61-65. doi: 10.1038/sj.gene.6363630

Verlaan, D. J., Berlivet, S., Hunninghake, G. M., Madore, A. M., Larivière, M., Moussette, S., et al. (2009). Allele-specific chromatin remodeling in the ZPBP2/GSDMB/ORMDL3 locus associated with the risk of asthma and autoimmune disease. Am. J. Hum. Genet. 85, 377-393. doi: 10.1016/j.ajhg.2009. 08.007

Weiss, L. A., Kosova, G., Delahanty, R. J., Jiang, L., Cook, E. H., Ober, C., et al. (2006). Variation in ITGB3 is associated with whole-blood serotonin level and autism susceptibility. Eur. J. Hum. Genet. 14:923-931. doi: 10.1038/sj.ejhg.5201644

Weiss, L. A., Veenstra-Vanderweele, J., Newman, D. L., et al. (2004). Genomewide association study identifies ITGB3 as a QTL for whole blood serotonin. Eur. J. Hum. Genet. 12, 949-954. doi: 10.1038/sj.ejhg.5201239

Zhu, G., Yoshida, S., Migita, K., Yamada, J., Mori, F., Tomiyama, M., et al. (2012). Dysfunction of extrasynaptic GABAergic transmission in phospholipase C-related, but catalytically inactive protein 1 knockout mice is associated with an epilepsy phenotype. J. Pharmacol. Exp. Ther. 340, 520-528. doi: 10.1124/ jpet.111.182386

Conflict of Interest Statement: The Guest Associate Editor Mariza De Andrade declares that, despite having collaborated with authors Bahram Namjou, Joshua C. Denny, Leah C. Kottyan, Marylyn D. Ritchie, and Shefali S. Verma, the review process was handled objectively and no conflict of interest exists. The Review Editor Andrew Skol declares that, despite having collaborated with author John B. Harley, the review process was handled objectively and no conflict of interest exists. Marc E. Rothenberg is a consultant for Immune Pharmaceuticals and has an equity interest. Marc E. Rothenberg has a royalty interest in reslizumab being developed by Teva Pharmaceuticals. Marc E. Rothenberg, John B. Harley, and Leah C. Kottyan are co-inventors of a patent application, being submitted by CCHMC, concerning the genetics of EoE. The authors declare that the research was conducted in the absence of any commercial or financial relationships that could be construed as a potential conflict of interest.

Received: 29 May 2014; accepted: 31 October 2014; published online: 18 November 2014.

Citation: Namjou B, Marsolo K, Caroll RJ, Denny JC, Ritchie MD, Verma SS, Lingren T, Porollo A, Cobb BL, Perry C, Kottyan LC, Rothenberg ME, Thompson SD, Holm IA, Kohane IS and Harley JB (2014) Phenome-wide association study (PheWAS) in EMR-linked pediatric cohorts, genetically links PLCL1 to speech language development and IL5-IL13 to Eosinophilic Esophagitis. Front. Genet. 5:401. doi: 10.3389/fgene. 2014.00401

This article was submitted to Applied Genetic Epidemiology, a section of the journal Frontiers in Genetics.

Copyright $\odot 2014$ Namjou, Marsolo, Caroll, Denny, Ritchie, Verma, Lingren, Porollo, Cobb, Perry, Kottyan, Rothenberg, Thompson, Holm, Kohane and Harley. This is an open-access article distributed under the terms of the Creative Commons Attribution License (CC BY). The use, distribution or reproduction in other forums is permitted, provided the original author(s) or licensor are credited and that the original publication in this journal is cited, in accordance with accepted academic practice. No use, distribution or reproduction is permitted which does not comply with these terms. 